\title{
Diffusion-Weighted Zonal Oblique Multislice-EPI Enhances the Detection of Small Lesions with Diffusion Restriction in the Brain Stem and Hippocampus: A Clinical Report of Selected Cases
}

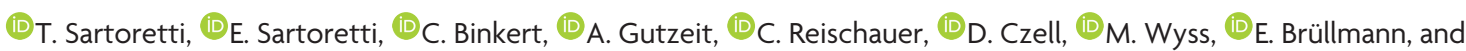 \\ (1)S. Sartoretti-Schefer
}

\begin{abstract}
SUMMARY: Diffusion restriction is the morphologic hallmark of acute ischemic infarcts and excitotoxic brain injury in various cerebral pathologies. Diffusion restriction is visible as hyperintensity on DWI and as hypointensity on ADC maps. Due to the vicinity of multiple anatomic structures in the brain stem and hippocampus, very small lesions with diffusion restriction may result in severe clinical symptomatology, but these small lesions easily go undetected on standard cerebral DWI due to insufficient spatial resolution, $\mathrm{T} 22^{*}$ blurring, and image artifacts caused by susceptibility-related image distortions. Diffusion-weighted zonal oblique multislice-EPI with reduced FOV acquisition permits a considerable increase in spatial resolution and enhances the visualization of very small pathologic lesions in the brain stem and hippocampus. Improved performance in the depiction of different pathologic lesions with diffusion restriction in the brain stem and hippocampus using this sequence compared with standard DWI in selected cases is presented.
\end{abstract}

ABBREVIATIONS: CN III = oculomotor nerve nucleus area; CN VI = abducens nerve nucleus area; DW = diffusion-weighted; sshot SE = single-shot spin-echo; $\mathrm{TGA}=$ transient global amnesia; ZOOM = zonal oblique multislice

D iffusion restriction is caused by different cerebral pathologies leading to cytotoxic edema by energy failure, altered intra- and extracellular ion and water concentrations, and excitotoxic brain injury due to extracellular increase of glutamate. ${ }^{1,2}$ These mechanisms may overlap in certain pathologies. Diffusion restriction is the main characteristic feature of an acute ischemic event ${ }^{2}$; therefore, DWI is the only reliable sequence for detecting an acute ischemic event.

DWI is routinely used in brain protocols for the evaluation of various cerebral pathologies characterized by diffusion restriction. The limited spatial resolution of a standard single-shot spin-echo (sshot SE) EPI DWI, called "standard DWI" in our work, is an enormous disadvantage if the clinical symptomatology indicates a lesion in the brain stem and hippocampus.

This clinical report shows how diffusion-weighted (DW) zonal oblique multislice (ZOOM)-EPI can improve the detection of DWI abnormalities in pathologies in the brain stem and hippocampus by increasing spatial resolution, allowing the visualization of small lesions with diffusion restriction not visible on standard DWI.

Received October 19, 2017; accepted after revision March 5, 2018

From the Institut für Radiologie (T.S., E.S., C.B., M.W., S.S.-S.), Kantonsspital Winterthur, Winterthur, Switzerland; Institut für Radiologie und Nuklearmedizin (A.G., C.R.), Hirslanden Klinik St. Anna, Luzern, Switzerland; Medizinische Klinik (D.C.), Abteilung für Neurologie, Uznach, Switzerland; and Philips HealthSystems (M.W., E.B.), Zürich, Switzerland.

Please address correspondence to Sabine Sartoretti-Schefer, MD, Institut für Radiologie, Kantonsspital Winterthur, Brauerstr 15, 8401 Winterthur, Switzerland; e-mail: sabine.sartoretti@ksw.ch

http://dx.doi.org/10.3174/ajnr.A5635

\section{MATERIALS AND METHODS}

In this clinical report, the MR images of 7 patients of a group with 44 patients ( 23 women and 21 men; $40-89$ years of age; mean age, 76.4 years) are depicted. Informed and written consent for publication of patient data and images was obtained by all patients described in this study. All patients with various acute clinical symptoms all indicating a brain stem pathology (mainly characterized by nausea, vertigo, ataxia, nystagmus, gaze disorders) or hippocampal pathology with amnestic syndrome were examined by both standard transverse sshot SE EPI DWI (called "standard DWI") and transverse DW ZOOM-EPI during the same examination. The acquisition of DW ZOOM-EPI followed immediately after the acquisition of the standard DWI on the same MR imaging scanner with an identical field strength. Identical image plane angulation was used in both transverse diffusion-weighted sequences.

Patient examinations were performed from October 2016 to August 2017 on a $1.5 \mathrm{~T}$ Ingenia (Philips Healthcare, Best, the Netherlands) or a 3T Achieva (Philips Healthcare) scanner. Imaging parameters of DW ZOOM-EPI and of standard DWI are shown in Tables 1 and 2. In all patients, 3D FLAIR imaging and transverse T2 TSE sequences were added for possible confirmation of the suspected pathology.

In each patient, the presence or absence of acute ischemic lesions in the brain stem on standard DWI and on DW ZOOMEPI was determined retrospectively. In 27 patients, no pathologic lesion was visible on both standard DWI and DW 


\begin{tabular}{lcc}
\hline & $\begin{array}{c}\text { DW ZOOM-EPI } \\
\text { on 1.5T MRI }\end{array}$ & $\begin{array}{c}\text { DW ZOOM-EPI } \\
\text { on 3T MRI }\end{array}$ \\
\hline Sequence type & sshot SE-EPI & sshot SE-EPI \\
Slice orientation & Transversal & Transversal \\
FOV (RL $\times$ AP) & $97 \times 92 \mathrm{~mm}^{2}$ & $180 \times 64 \mathrm{~mm}^{2}$ \\
Acquisition voxel size (RL $\times \mathrm{AP} \times \mathrm{FH})$ & $1.2 \times 1.2 \times 3.0 \mathrm{~mm}^{3}$ & $1.0 \times 1.15 \times 3.0 \mathrm{~mm}^{3}$ \\
No. of slices & 19 & 25 \\
TR & $3545 \mathrm{~ms}$ & $2899 \mathrm{~ms}$ \\
TE & $85 \mathrm{~ms}$ & $64 \mathrm{~ms}$ \\
Flip angle & $90^{\circ}$ & $90^{\circ}$ \\
Half scan factor & 0.6 & 0.6 \\
Bandwidth & $11.5 \mathrm{~Hz} / \mathrm{pixel}$ & $12.9 \mathrm{~Hz} / \mathrm{pixel}$ \\
EPI factor & 75 & 81 \\
No. of signal averages & 5 & 2 \\
Acquisition duration (minutes:seconds) & $05: 39$ & $03: 04$ \\
Fat suppression & $\mathrm{SPAIR}$ & $\mathrm{SPIR}$ \\
b-values & $0,1000 \mathrm{~s} / \mathrm{mm}^{2}$ & $0,1000 \mathrm{~s} / \mathrm{mm}^{2}$ \\
\hline
\end{tabular}

Note:-SPIR indicates spectral presaturation with inversion recovery; SPAIR, spectral attenuated inversion recovery; $\mathrm{RL}$, right left; AP, anterior posterior; FH, feet head.

Table 2: Imaging parameters of standard DWI

\begin{tabular}{lcc}
\hline & $\begin{array}{c}\text { Standard DWI } \\
\text { on } 1.5 T \text { MRI }\end{array}$ & $\begin{array}{c}\text { Standard DWI } \\
\text { on 3T MRI }\end{array}$ \\
\hline Sequence type & sshot SE-EPI & sshot SE-EPI \\
Slice orientation & Transversal & Transversal \\
FOV (RL $\times$ AP) & $230 \times 230 \mathrm{~mm}^{2}$ & $230 \times 230 \mathrm{~mm}^{2}$ \\
Acquisition voxel size (RL $\times$ AP $\times \mathrm{FH})$ & $1.4 \times 2.0 \times 3.0 \mathrm{~mm}^{3}$ & $1.55 \times 1.95 \times 3.0 \mathrm{~mm}^{3}$ \\
No. of slices & 47 & 50 \\
TR & $6642 \mathrm{~ms}$ & $6224 \mathrm{~ms}$ \\
TE & $97 \mathrm{~ms}$ & $85 \mathrm{~ms}$ \\
Flip angle & $90^{\circ}$ & $90^{\circ}$ \\
Sensitivity encoding factor & 2 & 2 \\
Half scan factor & 0.8 & $\mathrm{No}$ \\
Bandwidth & $16.6 \mathrm{~Hz} / \mathrm{pixel}$ & $20.7 \mathrm{~Hz} / \mathrm{pixel}$ \\
EPI factor & 57 & 59 \\
No. of signal averages & 1 & 1 \\
Acquisition duration (minutes:seconds) & $02: 16$ & $01: 52$ \\
Fat suppression & $\mathrm{SPIR}$ & $\mathrm{SPIR}$ \\
b-values & $0,1000 \mathrm{~s} / \mathrm{mm}^{2}$ & $0,1000 \mathrm{~s} / \mathrm{mm}^{2}$ \\
\hline
\end{tabular}

Note:-SPIR indicates spectral presaturation with inversion recovery; RL, right left; AP, anterior posterior; FH, feet head.
Hippocampal Ischemia. An 83-year-old woman with atrial fibrillation presented with acute hemiparesis on the left side and antegrade amnesia (Fig 2). Multiple punctate acute embolic ischemic infarcts with diffusion restriction were visible on DW ZOOM-EPI with ADC maps, namely in the mesencephalon along the pyramidal tract, in the left medial occipitotemporal gyrus, in the left hippocampus, and bilaterally in the preand postcentral area on the right. The punctate hippocampal infarct was not visible on standard DWI or FLAIR.

Acute Internuclear Ophthalmoplegia and Partial Oculomotor Nerve Palsy. A 44-year-old woman presented with acute internuclear ophthalmoplegia and a partial oculomotor nerve palsy on the left. The acute ischemic infarct in the oculomotor nerve nuclear area (CN III) and the medial longitudinal fascicle was visible on DW ZOOM-EPI and ADC maps but not on standard DW and was only faintly apparent on FLAIR (Fig 3). The ischemic infarct was caused by an acute embolus from a pulmonary arteriovenous malformation.

Acute Internuclear Ophthalmoplegia and Acute Abducens Palsy. A 71-year-old man presented with acute internuclear ophthalmoplegia/abducens nerve palsy of the left side, skew deviation, and nystagmus to the right side. An acute ischemic infarct with diffusion restriction was visi-
ZOOM-EPI. In 9 patients both standard DWI and DW ZOOM-EPI showed an acute ischemic infarct with diffusion restriction, involving both the brain stem and cerebellum in 6 patients, only the cerebellum in 2 patients, and only the brain stem in 1 patient. All infarcts visible on both standard DWI and DW ZOOMEPI had a minimum size of $1 \mathrm{~cm}$. In 8 patients, mostly punctate acute ischemic foci, either in the brain stem, cerebellum, or hippocampi, were only visible on DW ZOOM-EPI.

Despite the acquisition time of the DW ZOOM-EPI (3 minutes to 5 minutes and 30 seconds), no visually evident movement artifacts in the patients examined were observed, but a quantification of movement artifacts was not performed.

\section{Case Series}

Transient Global Amnesia. A 65-year-old female patient presented with antegrade and retrograde amnesia due to clinically suspected transient global amnesia (TGA) lasting for 18 hours (Fig 1). Punctate diffusion restriction was demonstrated on DW ZOOM-EPI with ADC maps in the lateral hippocampal body as a specific TGA-associated lesion. No abnormality was visible on standard DWI or on FLAIR. ble on DW ZOOM-EPI and ADC maps involving the left medial longitudinal fascicle and the abducens nerve nuclear area $(\mathrm{CN}$ VI). Standard DWI and FLAIR images did not show any abnormality (Fig 4).

Acute Ischemic Infarct in the Left Inferior Cerebellar Peduncle. A 57 -year-old woman presented with severe intractable vertigo, nystagmus, and vomiting due to isolated vestibular syndrome lasting 3 days. A punctate diffusion abnormality on DW ZOOMEPI and ADC maps (Fig 5) in the left inferior cerebellar peduncle was present, not visible on standard DWI or FLAIR.

Acute Ischemic Infarct in the Right Nucleus Prepositus Hypoglossi. A 40-year-old female patient presented with acute forward and backward vertigo and horizontal nystagmus. A punctate diffusion abnormality was visible on DW ZOOM-EPI and ADC maps (Fig 6) in the right nucleus prepositus hypoglossi; the lesion was not depicted on standard DWI and on FLAIR.

Acute Ischemic Infarct in the Right Flocculus. A 51-year-old woman presented with vertigo and saccadic eye movements during the last 5 days. A punctate diffusion abnormality representing her infarction 


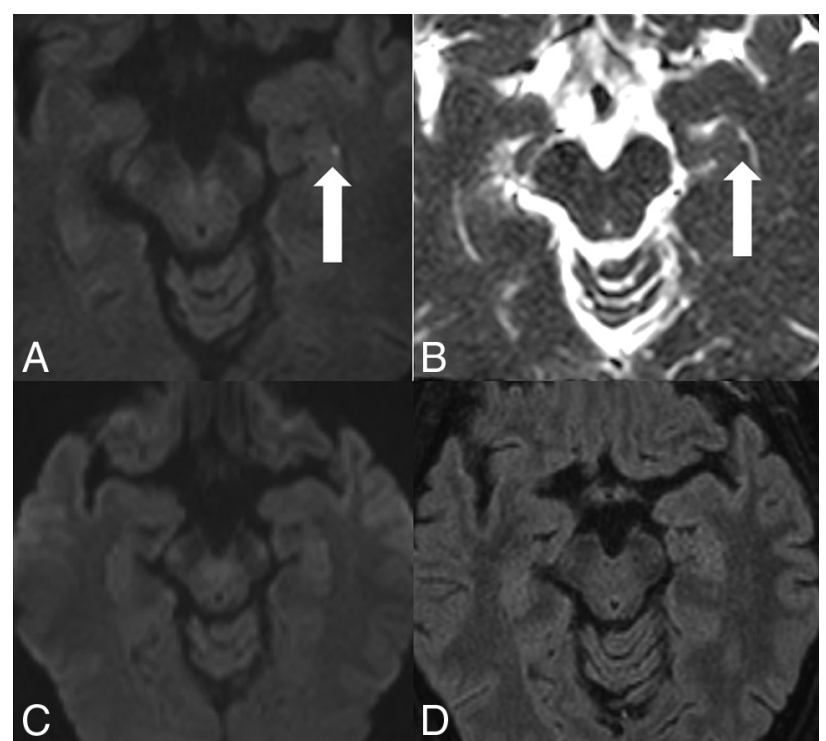

FIG 1. TGA. MR imaging was performed on a 1.5T scanner 24 hours after the beginning of the clinical symptomatology. On DWI ZOOMEPI (white arrow in A). a punctate hyperintensity and, on DWI ZOOMEPI ADC map (white arrow in $B$ ), a punctate hypointensity with an ADC of $0.915 \times 10^{-3} \mathrm{~mm}^{2} /$ second were demonstrated in the left lateral hippocampal body as a specific TGA-associated lesion. ADC in the normal right hippocampus was $1.093 \times 10^{-3} \mathrm{~mm}^{2} / \mathrm{second}$. Standard DWI (C) did not show the abnormality nor was any signal intensity change visible on transverse FLAIR $(D)$.

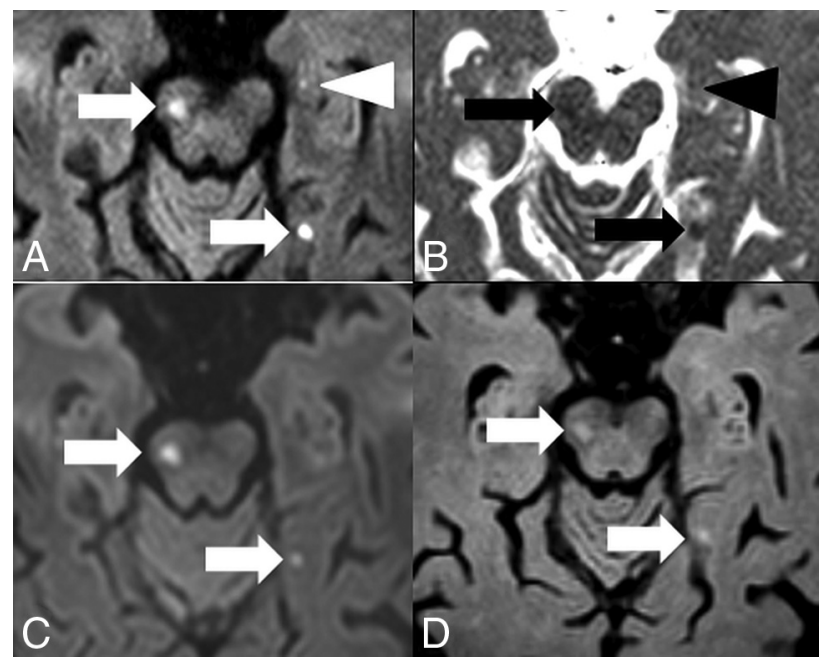

FIG 2. Hippocampal embolic ischemia. MR imaging was performed on a $1.5 T$ scanner. On DW ZOOM-EPI, 3 acute ischemic infarcts in the right mesencephalon along the pyramidal tract (white arrow), in the left medial occipitotemporal gyrus (white arrow), and in the left hippocampus (white arrowhead) were visible as hyperintense foci in A. On DW ZOOMEPI ADC, the same infarcts were hypointense in $B$ marked with black arrows and a black arrowhead (with an ADC of $0.817 \times 10^{-3} \mathrm{~mm}^{2}$ / second compared with the ADC of $1.03 \times 10^{-3} \mathrm{~mm}^{2} / \mathrm{second}$ in the normal right hippocampus). The punctate small hippocampal infarct on the left side was not visible on standard DWI (C) or FLAIR (D).

was present in the right flocculus on DW ZOOM-EPI and ADC maps (Fig 7). Standard DWI and FLAIR findings were normal.

\section{DISCUSSION}

Standard sshot SE EPI DWI is prone to geometric distortions, mainly due to the long readout time and low bandwidth in the

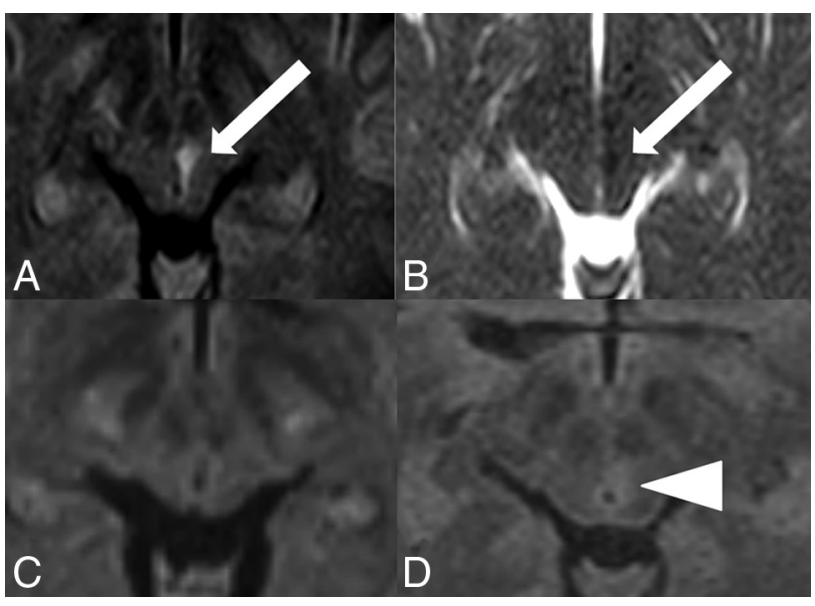

FIG 3. Acute internuclear ophthalmoplegia with partial oculomotor nerve palsy. MR imaging was performed on a $3 \mathrm{~T}$ scanner. The acute ischemic infarct in left CN III and the medial longitudinal fascicle was visible as a hyperintensity (white arrow) on DW ZOOM-EPI $(A)$, as a hypointensity (white arrow) on DW ZOOM-EPI ADC (B) with an ADC of $0.6 \times 10^{-3} \mathrm{~mm}^{2} /$ second (compared with an ADC of $0.985 \times 10^{-3}$ $\mathrm{mm}^{2} /$ second in the normal right CN III), and as very faintly hyperintense on transverse FLAIR ( $D$, white arrowhead). No pathologic findings were seen on standard DWI (C).

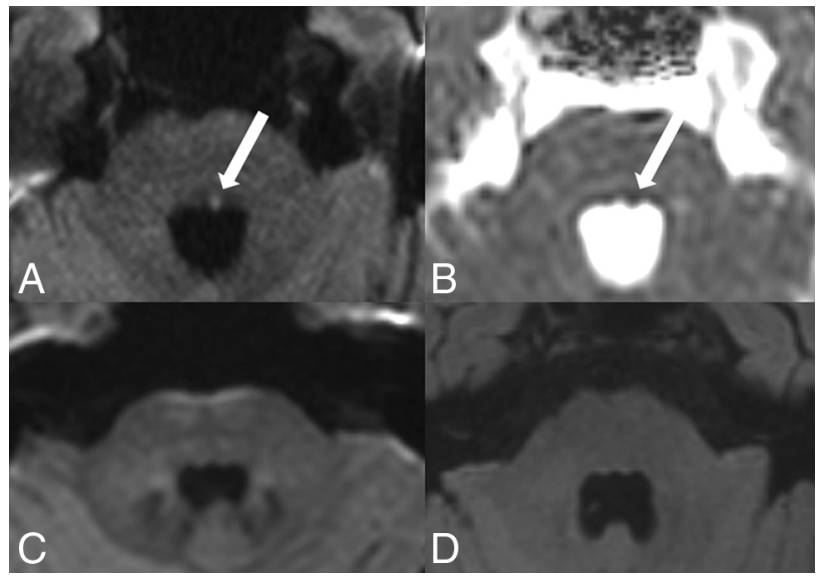

FIG 4. Acute internuclear ophthalmoplegia with abducens nerve palsy. MR imaging was performed on a 3T scanner. The punctate acute ischemic infarct in the left CN VI and the medial longitudinal fascicle, located in the inferior pons, was visible as hyperintensity (white arrow) on DW ZOOM-EPI (A) and as hypointensity (white arrow) on DW ZOOM-EPI ADC with an ADC of $0.707 \times 10^{-3} \mathrm{~mm}^{2} /$ second $(B)$ but was not visible on standard DWI (C, with slightly different angulation compared with DW ZOOM-EPI) and on FLAIR (D). ADC in the right CN $\mathrm{VI}$ area was $0.948 \times 10^{-3} \mathrm{~mm}^{2} /$ second. A local microangiopathic origin was suspected. No cardiovascular embolic source was found.

phase-encoding direction. Reduced-FOV imaging allows acquisition of a small FOV with either reduced geometric distortion (shorter readout time) or higher spatial resolution (same readout time) compared with standard EPI DWI. An FOV smaller than the object typically induces foldover artifacts. Numerous different techniques have been introduced in the past to prevent these, especially selective excitation methods that excite only the ROI such as "inner volume imaging" and suppression-based methods that apply bands to saturate signal external to the target FOV such as "outer volume suppression" or a combination of both..$^{3-7}$

Reduced-FOV imaging or ZOOM imaging or small-FOV imaging is a commonly used technique in the context of data acqui- 


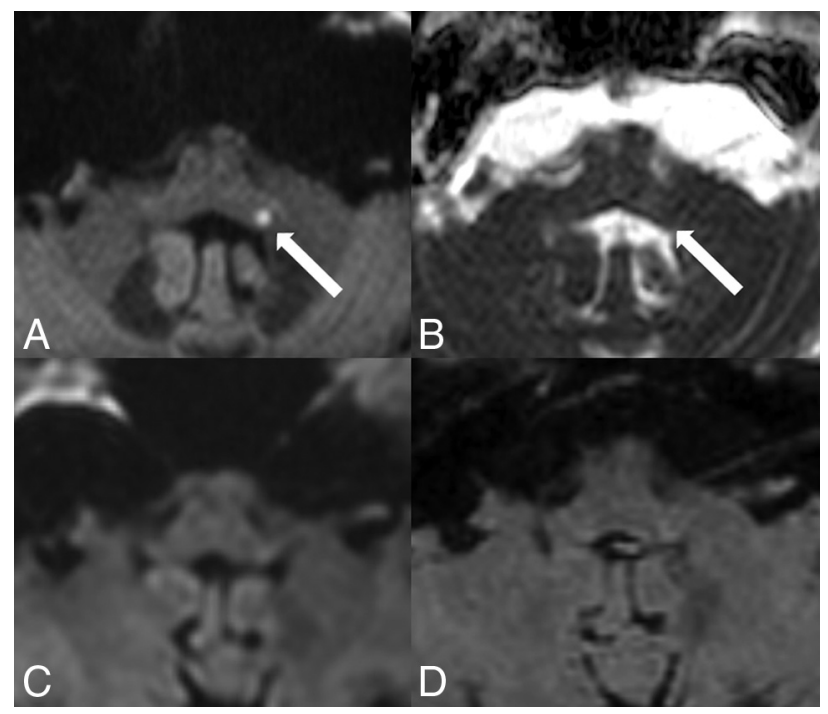

FIG 5. Acute ischemic infarct in the left inferior cerebellar peduncle. MR imaging was performed on a 3T scanner. Note a punctate area with hyperintensity in the inferior cerebellar peduncle on the left side on DW ZOOM-EPI (white arrow in A) and as a slight hypointensity on DW ZOOM-EPI ADC with an ADC of $0.562 \times 10^{-3} \mathrm{~mm}^{2} /$ second (white arrow in B) compared with the ADC of $0.841 \times 10^{-3} \mathrm{~mm}^{2}$ / second in the normal right inferior cerebellar peduncle. This diffusion abnormality was not visible on transverse standard DWI (C) or the transverse FLAIR image $(D)$. Severe chronic microangiopathy was present in the supra- and infratentorial areas.

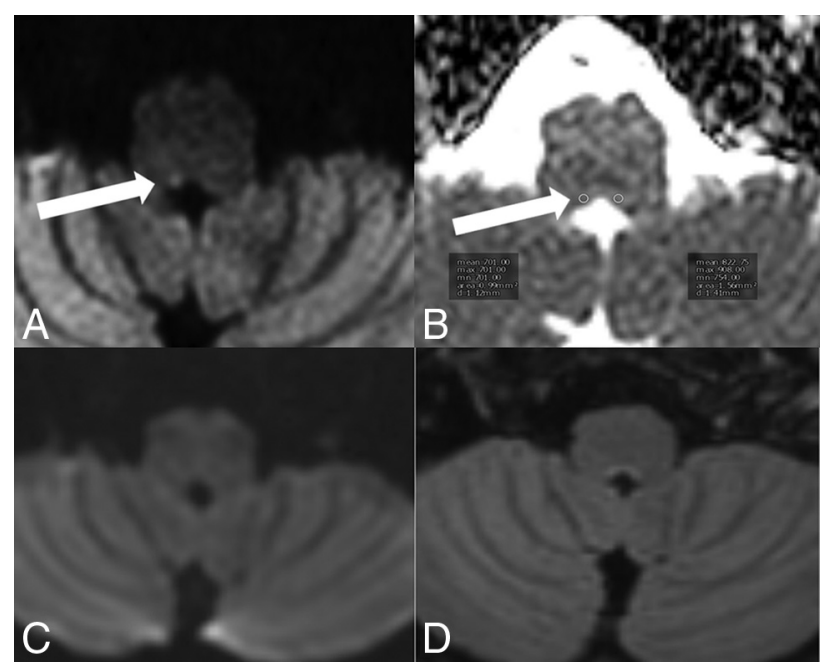

FIG 6. Acute ischemic infarct in the nucleus prepositus hypoglossi on the right side. MR imaging was performed on a 1.5T scanner. A punctate area with diffusion restriction was visible in the right nucleus prepositus hypoglossi. This lesion was hyperintense on DW ZOOMEPI (white arrow in A) and slightly hypointense on DW ZOOM-EPI $A D C$ with an ADC of $0.701 \times 10^{-3} \mathrm{~mm}^{2} /$ second (white arrow in $B$ ) compared with an ADC of $0.822 \times 10^{-3} \mathrm{~mm}^{2} /$ second in the normal left nucleus prepositus hypoglossi. The lesion was not visible on transverse standard DWI $(C)$ and FLAIR $(D)$ and was thought to be of local microangiopathic origin.

sition of small FOVs within larger objects. In this work, a noncoplanar excitation combined with outer volume suppression, originally presented by Wilm et $\mathrm{al},{ }^{8}$ was used. This technique is now referred to as DW ZOOM-EPI, and the main applications using small-FOV imaging are imaging of the prostate, spinal cord, pancreas, breast, and heart, ${ }^{5-11}$ where a relatively small

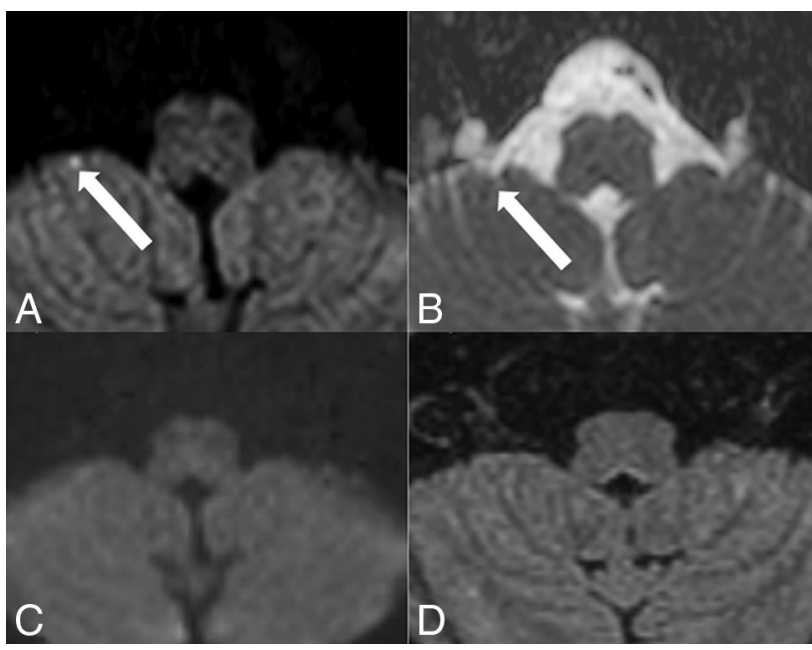

FIG 7. Subacute ischemic infarct in the right flocculus. MR imaging was performed on a 1.5T scanner. On DW ZOOM-EPI, a punctate hyperintensity (white arrow, $A$ ) with isointensity on DW ZOOM-EPI $A D C$ and an ADC of $0.878 \times 10^{-3} \mathrm{~mm}^{2} /$ second (white arrow, B) represents a subacute ischemic infarct in the right flocculus. In the normal left flocculus, the ADC was $0.926 \times 10^{-3} \mathrm{~mm}^{2} /$ second. Standard DWI $(C)$ and FLAIR $(D)$ findings were normal. The lesion was thought to be of local microangiopathic origin.

area of interest surrounded by tissue of less interest is depicted with high resolution. However, to date, the use of reducedFOV imaging in the depiction of very small pathologic lesions with diffusion restriction in the brain stem and hippocampus has not yet been systematically evaluated. ${ }^{12-18}$ The imaging examples presented in this clinical report stress but do not prove the importance of reduced-FOV imaging for the visualization of small lesions in the brain stem and hippocampus.

The increased detectability of small lesions with diffusion restriction in the brain stem and hippocampus on DW ZOOM-EPI in comparison with standard DWI might have been influenced by several technical factors:

1) The registration effect: The standard DWI and the DW ZOOM-EPI slice selections may be slightly different. As a result, the comparison of visual lesion detectability may not entirely represent an effect of the sequence in these patients.

2) The difference in scan time: The total scan time and thus the signal-to-noise ratio differed between standard DWI and DW ZOOM-EPI. The increased scan time in DW ZOOM-EPI automatically increased the signal-to-noise ratio and could also affect the visibility of lesions.

3) The difference in the display window/level settings: Due to the differences in the imaging parameters between standard DWI and DW ZOOM-EPI, it was not possible to keep an identical display window/level. Window and level selection was at the discretion of the reviewer to optimally depict pathology.

Therefore, a next step might be a prospective study of standard DWI and DW ZOOM-EPI with constant imaging parameters to make a precise comparison of the 2 sequences. This would help clarify whether the improved visibility of small lesions originates from a higher SNR, higher resolution, reduced image distortion, or a combination of these factors. 


\section{CONCLUSIONS}

Pathologies with diffusion restriction are routinely depicted by standard DWI. However, the visualization of very small lesions with diffusion restriction in the brain stem and hippocampus may be difficult on standard DWI. Tailored DW ZOOM-EPI enhances the visualization of very small lesions in the brain stem and hippocampus and thus can be the only MR image depicting the pathology. The acquisition of DW ZOOM-EPI may be recommended in selected patients presenting with specific brain stem syndromes or with clinical suspicion of hippocampal pathologies such as TGA or hippocampal ischemia and with negative standard DWI findings. The correct diagnosis of an ischemic-pathologic lesion can expedite correct subsequent therapy.

\section{ACKNOWLEDGMENTS}

The authors thank Dr Simon Rauch, Institute of Radiology, Kantonsspital Winterthur, Winterthur, Switzerland, for his support in the preparation of the manuscript.

Disclosures: Christoph Binkert—UNRELATED: Consultancy: Merit Medical Systems, Biotronik; Grants/Grants Pending: Cook, Merit Medical Systems. David CzellUNRELATED: Payment for Development of Educational Presentations: University of Zürich. Michael Wyss - UNRELATED: Employment: My position in the hospital is paid by Philips within the framework of a collaboration agreement, Comments: $€ 4000$ per year.* Erika Brüllmann—OTHER RELATIONSHIPS: Philips HealthSystems employee. *Money paid to the institution.

\section{REFERENCES}

1. Moritani T, Smoker WRK, Sato Y, et al. Diffusion-weighted imaging of acute excitotoxic brain injury. AJNR Am J Neuroradiol 2005;26: 216-28 Medline

2. Schaefer PW, Grant PE, Gonzalez RG. Diffusion-weighted MRI imaging of the brain. Radiology 2000;217:331-45 CrossRef Medline

3. Wargo CJ, Moore J, Gore JC. A comparison and evaluation of reduced-FOV methods for multi-slice 7T human imaging. Magn Reson Imaging 2013;31:1349-59 CrossRef Medline

4. Wheeler-Kingshott CA, Parker GJ, Symms MR, et al. ADC mapping of the human optic nerve: increased resolution, coverage, and reliability with CSF-suppressed ZOOM-EPI. Magn Reson Med 2002;47: 24-31 CrossRef Medline
5. Andre JB, Bammer R. Advanced diffusion-weighted magnetic resonance imaging techniques of the human spinal cord. Top Magn Reson Imaging 2010;21:367-78 CrossRef Medline

6. Dowell NG, Jenkins TM, Ciccarelli O, et al. Contiguous-slice zonally oblique multislice (COZOOM) diffusion tensor imaging: examples of in vivo spinal cord and optic nerve applications. J Magn Reson Imaging 2009;29:454-60 CrossRef Medline

7. Samson RS, Lévy S, Schneider T, et al. ZOOM or non-ZOOM? Assessing spinal cord diffusion tensor imaging protocols for multicentre studies. PLoS One 2016;11:e0155557 CrossRef Medline

8. Wilm BJ, Svensson J, Henning A, et al. Reduced field-of-view MRI using outer volume suppression for spinal cord diffusion imaging. Magn Reson Med 2007;57:625-30 CrossRef Medline

9. Dong H, Li Y, Li H, et al. Study of the reduced field-of-view diffusion-weighted imaging of the breast. Clin Breast Cancer 2014;14: 265-71 CrossRef Medline

10. Kim H, Lee JM, Yoon JH, et al. Reduced field-of-view diffusionweighted magnetic resonance imaging of the pancreas: comparison with conventional single-shot echo-planar imaging. Korean J Radiol 2015;16:1216-25 CrossRef Medline

11. Reischauer C, Wilm BJ, Froehlich JM, et al. High-resolution diffusion tensor imaging of prostate cancer using a reduced FOV technique. Eur J Radiol 2011;80:34-41 CrossRef Medline

12. Förster A, Griebe M, Gass A, et al. Diffusion-weighted imaging for the differential diagnosis of disorders affecting the hippocampus. Cerebrovasc Dis 2012;33:104-15 CrossRef Medline

13. Bartsch T, Deuschl G. Transient global amnesia: functional anatomy and clinical implications. Lancet Neurol 2010;9:205-14 CrossRef Medline

14. Weon YC, Kim JH, Lee JS, et al. Optimal diffusion-weighted imaging protocol for lesion detection in transient global amnesia. AJNR Am J Neuroradiol 2008;29:1324-28 CrossRef Medline

15. Dekezyer S, De Kock S, Nikoubashman O, et al. "Unforgettable": a pictorial essay on anatomy and pathology of the hippocampus. Insights Imaging 2017;8:199-212 CrossRef Medline

16. Szabo K, Förster A, Jäger Th, et al. Hippocampal lesion patterns in acute posterior cerebral artery stroke: clinical and MRI findings. Stroke 2009;40:2042-45 CrossRef Medline

17. Kim HJ, Lee $\mathrm{SH}$, Park JH, et al. Isolated vestibular nuclear infarction: report of two cases and review of the literature. J Neurol 2014;261:121-29 CrossRef Medline

18. Kim SH, Park SH, Kim HJ, et al. Isolated central vestibular syndrome. Ann N.Y. Acad Sci 2015;1343:2015:80-89 CrossRef Medline 